\title{
Exploration on Gleason score variation trend of patients with prostate carcinoma from 1996 to 2019: a retrospective single center study
}

\author{
Weiyu Zhang ${ }^{1 \# \wedge}$, Gongwei Wang ${ }^{2 \#}$, Fengling $\operatorname{Lan}^{3}$, Huanrui Wang ${ }^{1}$, Danhua Shen ${ }^{2}$, Kexin Xu ${ }^{1}$, Tao Xu ${ }^{1}$, \\ $\mathrm{Hao} \mathrm{Hu}^{1 \wedge}$ \\ ${ }^{1}$ Department of Urology, Peking University People's Hospital, Beijing, China; ${ }^{2}$ Department of Pathology, Peking University People’s Hospital, \\ Beijing, China; ${ }^{3}$ Department of Human Resources, Peking University People's Hospital, Beijing, China \\ Contributions: (I) Conception and design: W Zhang, G Wang; (II) Administrative support: G Wang, H Hu; (III) Provision of study materials or \\ patients: K Xu, D Shen; (IV) Collection and assembly of data: H Wang, F Lan; (V) Data analysis and interpretation: F Lan, H Wang; (VI) Manuscript \\ writing: All authors; (VII) Final approval of manuscript: All authors. \\ "These authors contributed equally to this work. \\ Correspondence to: Hao Hu. Peking University People's Hospital, No. 11 Xi Zhi Men South Street, Beijing 100044, China. Email: huhao@bjmu.edu.cn.
}

\begin{abstract}
Backgroundk Gleason score (GS) is one of the stronger prognostic factors and is integral to the management of prostate carcinoma. Subsequent modifications, recommended by the International Society of Urological Pathology in 2005 and 2014, enabled accurate prediction of prognosis. The present study investigated GS variation trend of patients with prostate carcinoma from 1996 to 2019 and offered an overview of GS changes with age, specimen type, histopathological type and serum prostate specific antigen
\end{abstract} (PSA).

Methods: One thousand three hundred and seventy-six patients, admitted to Peking University People's Hospital in 1996 to 2019, were divided into 1996 to 2006, 2007 to 2015 and 2016 to 2019 groups. Data, including demographic characteristics, GS, primary and secondary grade and percentage of primary and secondary grade of each group, were collected and analyzed. The population distribution and average of GS was evaluated, after segmented and stratified by age, type of specimen, histopathological type and PSA.

Results: The average of age and PSA of each cohort had no obvious change. The average of total GS fluctuated among three cohorts with statistically significant differences. The distribution of age and PSA did not differ among cohorts. The distribution of total and primary GS shifted, with more patients detected as total GS higher than 6 (86.1\%), and more primary grade higher than $3(56.7 \%)$ in 2016-2019. After segmented and stratified by age, specimen type, histological type and PSA, the population percentage of GS over 6 was significantly higher in 2016-2019 than 1996-2006 and 2007-2015 in patients aged younger than 80 years (age $<60,89.6 \%$, age 60-69, 82.0\%, age 70-79, 87.7\%). Patients, aged below 80 years in 2016-2019, were detected with higher total GS.

Conclusions: In the present study, GS in patients with prostate carcinoma showed a upward trend. Primary grade, age, serum PSA and specimen type were the main reasons for GS changing while secondary grade, tissue types and diagnostic criteria influenced less.

Keywords: Prostate carcinoma (PCa); Gleason score; the type of specimen; age; prostate specific antigen (PSA)

Submitted Aug 07, 2020. Accepted for publication Dec 13, 2020.

doi: 10.21037 /gs-20-659

View this article at: http://dx.doi.org/10.21037/gs-20-659

^ ORCID: Weiyu Zhang 0000-0003-1134-4362; Hao Hu 0000-0002-8569-9679. 


\section{Introduction}

Prostate carcinoma (PCa) is the most commonly diagnosed solid-organ malignancy in men in China, according to data from the National Central Cancer Registry of China with an estimated 80,000 new prostate cancer in 2015 (1). Gleason grading system was born in 1966, and was one of considerable prognostic factors. The scoring system is based on the histological appearance of prostate cancer cells, consisted of a primary grade for the predominant histological pattern and a secondary grade for the highest pattern, both on a scale of 1 to 5 based on the glandular differentiation and the growth pattern in the stroma (2). The current system was modified in 2005 and 2014 by International Society of Urological Pathology (ISUP) $(2,3)$. As GS elevated, the prognosis got worsen (4-6). Pierorazio et al. (7) reported that the 5 -year survival percentage was $96.6 \%, 88.1 \%, 69.7 \%, 63.7 \%$ and $34.5 \%$ respectively for GS $\leq 6,3+4,4+3,8$ and 9-10. For now, GS is still an important indicator for PCa's prognosis.

In 2001, Gilliland et al. (8) proposed that there was a small but statistically rising trend of GS. In 2003, Chism et al. (9) found a statistically increases in GS. And in 2009, Zareba et al. (10) confirmed that there was a shift towards higher GS on biopsy and prostatectomy. In 2015, Danneman et al. (11) also reported there has been a gradual shift towards higher Gleason grading, which started before 2005 but became more evident after the ISUP 2005 revision. The literatures mentioned above presented an increased trend of GS over time. Chang in the early performed in decrease of 2-5 GS, which caused by over-diagnosis. In addition, change in type of specimen may lead to change of GS. Incidental prostate cancer was detected in all populations by mainly transurethral resection of prostate (TURP), with no obvious time trends in prevalence but lower percentage in all PCa specimen (12). GS of TURP specimen was lower and reduce of its quantity resulted in increase of GS. Modify of diagnostic criteria also leaded to GS shift $(13,14)$. Besides, age (15), histopathological type (16) and serum prostate specific antigen (PSA) (17) were relevant to GS. Physicians in China have applied Gleason Score system in diagnosis of PCa for years. There was no individual study on whether GS in China changed or existed the same changing trend as above. And the reasons for GS change remained unclear. Thus, it was necessary to carry out study to explore the PCa GS change trend of Chinese population.

We present the following article in accordance with the
STROBE reporting checklist (available at http://dx.doi. org/10.21037/gs-20-659).

\section{Methods}

\section{Study design}

The present study analyzed retrospectively all cases of prostate carcinoma diagnosed in Peking University People's Hospital over a period of 23 years (from January 1996 to December 2019). The study was conducted in accordance with the Declaration of Helsinki (as revised in 2013). This study was approved by Ethics Committee of Peking University People's Hospital (No. 2017PHB143) and performed in line with international ethics norms. Written informed consent was waived for the nature of retrospective study.

\section{Patients and groups}

Diagnostic criteria of PCa was with reference to the 2016 WHO Classification of Tumors of the Urinary System and Male Genital Organs (18). Patients were eligible if they were histopathologically diagnosed with prostate carcinoma. The first guideline of urology in China was published in 2006. It was the first time that PSA was identified as a detection indicator nation-widely, influencing the diagnosis and monitoring of prostate cancer greatly, especially, the early detection. The Gleason score classification has undergone major modifications to reflect modern diagnostic and therapeutic practices in 2014 and the modified system was accepted by WHO and published in 2016. Thus, the patients were divided to three groups by the year of 2006 and 2015, namely the frontal group of 1996 to 2006, the middle group of 2007 to 2015 and the latter group of 2016 to 2019 .

\section{Data collection}

Data including age, histopathological type, serum PSA, type of specimen and GS were collected. Type of specimen was classified as needle biopsy (NB), transurethral resection of prostate (TURP), radical prostatectomy (RP). When one patient underwent RP after NB, the data of RP was enrolled and the data of NB was excluded. Histopathological type was categorized as classic acini carcinoma and other types (including atrophy, false proliferative, foam, signet ring cell and ductal carcinoma). Serum PSA nearest to operation was 
included.

Prior to analysis, all specimens were collected, all of the pathology analysis was reevaluated by two pathologists according to the 2015 ISUP guidelines. When the two pathologists didn't reach agreement and a third pathologist couldn't reconciles the result, the corresponding data was excluded.

\section{Statistical analysis}

The data were presented as means \pm standard deviation. Patients' demographic, clinical and tumor characteristics, the GS, primary and secondary grade were compared least significant difference (LSD), One-way Anova, and $\chi^{2}$ or Fisher's exact test for categorical variables. $\mathrm{P}$ values of $<0.05$ were considered statistically significant. Statistical analyses were performed using IBM SPSS version 19.0 (SPSS Inc., Chicago, IL, USA).

\section{Results}

A total of 1,376 patients with prostate adenocarcinoma were included. Histopathological data was obtained by NB of patients with abnormal serum PSA or prostatic tubercles, $\mathrm{RP}$ of patients diagnosed with $\mathrm{PCa}$, and TURP of patients with incidental prostate cancer. PCa patients, admitted to our center, accelerated year by year. The total score, primary and secondary grade of Gleason floated by year. Several peaks and valleys shifted effected by factors, such as the application of PSA, the publication of China Urology Association guidelines, the increasing medical examination. The result of Gleason variation trend was caused by many reasons (Figure S1). The average of age stayed stable, even though the national population aging. Slightly upward trend of serum PSA was observed. Incidental prostate carcinoma detected by TURP occurred every year with very low rate. The rate of RP climbed year by year with the rate of NB scaled down. The pathological pattern of prostate acinar carcinoma occupied over $90 \%$ even as time goes on (Figure 1).

The average of age and PSA of each cohort had no obvious difference. The average of total Gleason score was volatile among three cohorts with statistically significant differences $(\mathrm{P}=0.000)$. The distribution of age and PSA did not differ among cohorts. Proportion of RP increased significantly in 2016-2019 compare to 1996-2006 and $2007-2015(\mathrm{P}=0.000)$. The distribution of total and primary
Gleason score shifted, with more patients detected as total Gleason score higher than $6(86.1 \%, \mathrm{P}=0.002)$, and more primary grade higher than $3(56.7 \%, \mathrm{P}=0.015)$ in 2016 2019. The distribution of histopathological type and serum PSA stable unchanged (Table 1). For digging deep into the factor influencing the GS shift, data of age, specimen type, histopathological type and PSA was segmented and stratified. Then the population distribution in total Gleason score less or equal to 6 , equal to 7 and more or equal to 8 in three cohorts was analyzed. The population percentage of Gleason score over 6 was significantly higher in 2016-2019 than 1996-2006 and 2007-2015 in patients aged younger than 80 years (age $<60,89.6 \%, P=0.000$, age $60-69,82.0 \%$, $\mathrm{P}=0.005$, age $70-79,87.7 \%, \mathrm{P}=0.000)$. But patients aged over 80 years didn't show the feature of distribution shift. Divided by specimen type, histopathological type and PSA, the same distribution characters remained with significantly statistical difference. After segmented and stratified, larger population in the latter group occupied in high GS than the frontal and middle group (Table 2).

The average of GS, primary grade and secondary grade in three cohorts was analyzed after segmented and stratified by age, type of specimen histopathological type and PSA. Patients, aged below 80 years in 2016-2019, were detected with higher total Gleason score than 1996-2006 and 2007$2015(\mathrm{P}<0.05)$. Specimen from RP, in 2016-2019, showed an increasing total Gleason score with significantly difference, compared with 1996-2006 and 2007-2015 ( $\mathrm{P}=0.000)$, while specimen from NB and TURP didn't. The patients diagnosed with classic acini prostate carcinoma or at all level of serum PSA showed the same trend with significantly difference with significantly statistical difference (Table 3). Patients, aged between 70 and 79, in 2016-2019, were detected with higher primary grade than these others with significantly difference $(\mathrm{P}=0.000)$. Specimen from $\mathrm{NB}$ and RP in 2016-2019, were detected with higher primary grade than these others with significantly difference $(\mathrm{P}<0.05)$. The same trend was observed in patients diagnosed with classic acini prostate carcinoma or at all level of serum PSA (Table 4). Patients, aged below 80 years in 2016-2019, were detected with higher secondary score $(\mathrm{P}<0.05)$. Specimen from RP, in 2016-2019, showed an increasing secondary score with significantly difference, compared with 1996 2006 and 2007-2015 ( $\mathrm{P}=0.000)$, while specimen from NB and TURP didn't. The patients diagnosed with classic acini prostate carcinoma or at all level of serum PSA showed the same trend with significantly difference $(\mathrm{P}=0.000)$ (Table 5). 

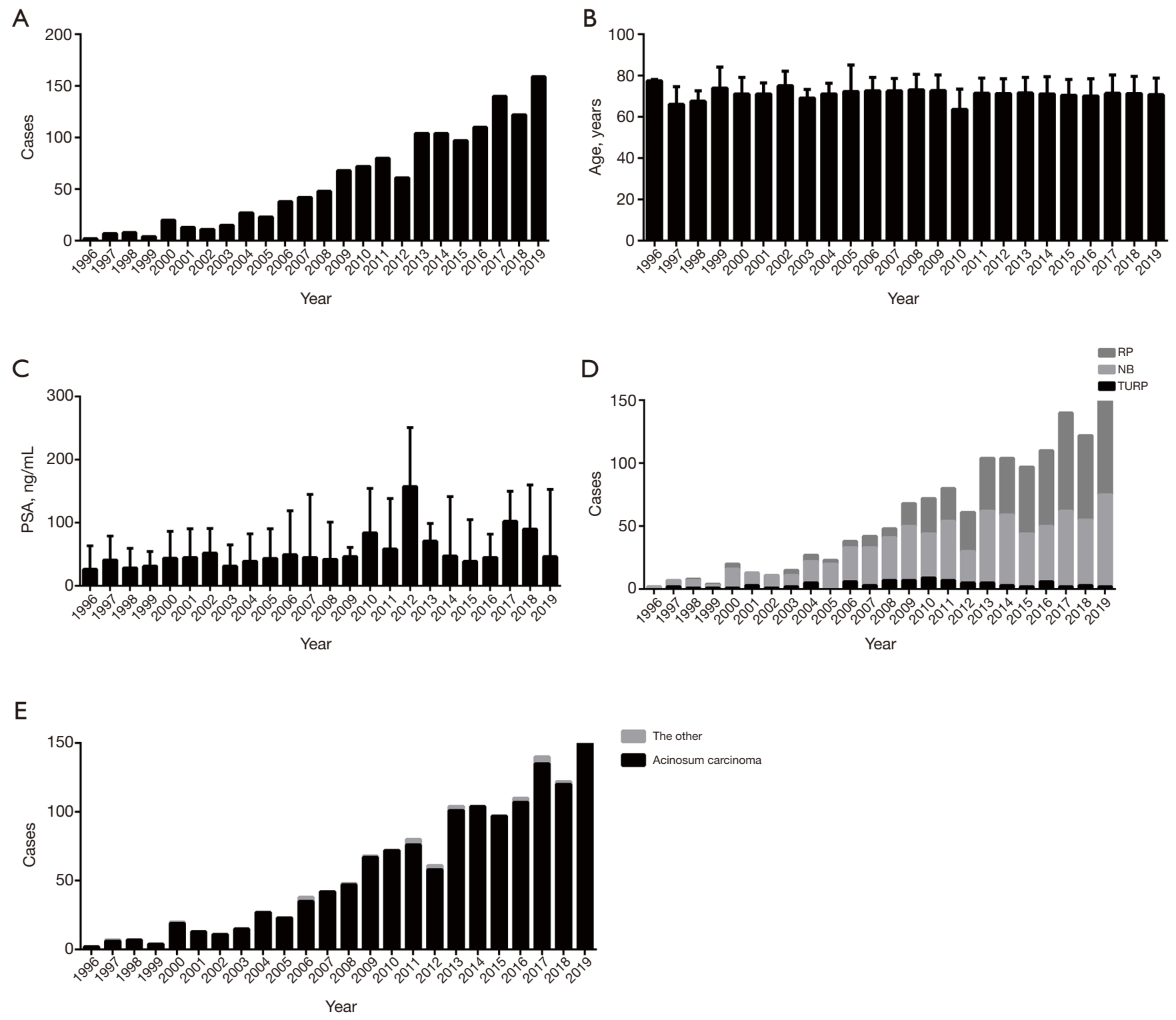

Figure 1 The overview of the PCa population. PCa patients accelerated year by year (A). The average of age stayed stable, even though the national population aging (B). Elevated serum PSA was observed (C). Incidental prostate carcinoma detected by TURP occurred every year with very low rate. The rate of radical prostatectomy climbed year by year with the rate of needle biopsy scaled down (D). The pathological pattern of prostate acinar carcinoma occupied over $90 \%$ even as time goes on (E).

\section{Discussion}

Current researches have proved an upward trend of GS. Chism et al. (9) developed a nomogram that percentage of GS 6 in 1992-1994 decreased significantly compared with 1996-1997 while percentage of GS 7 increased significantly. Zareba et al. (10) found that average of NB GS increased from 6.34 in 2000-2004 to 6.49 in 2005-2007, and RP increased from 6.58 to 6.78. In 2015, Danneman et al. (11) reported percentage of GS 7-10 in low-risk cancer (T1c and S-PSA $4-10 \mathrm{ng} / \mathrm{mL}$ ) increased from $16 \%$ in 1998 to $40 \%$ in 2011, and before 2005 it was $19 \%$ while after 2005 $33 \%$. The same situation was in high-risk cancer (T3 and S-PSA $20-50 \mathrm{ng} / \mathrm{mL}$ ). Percentage of GS 2-5 decreased from $27 \%$ in 1998 to $1 \%$ in 2011, and percentage of GS $2-4$ almost disappeared. In our research, percentage of GS $\leq 6$ 
Table 1 Basic information of PCa in three cohorts

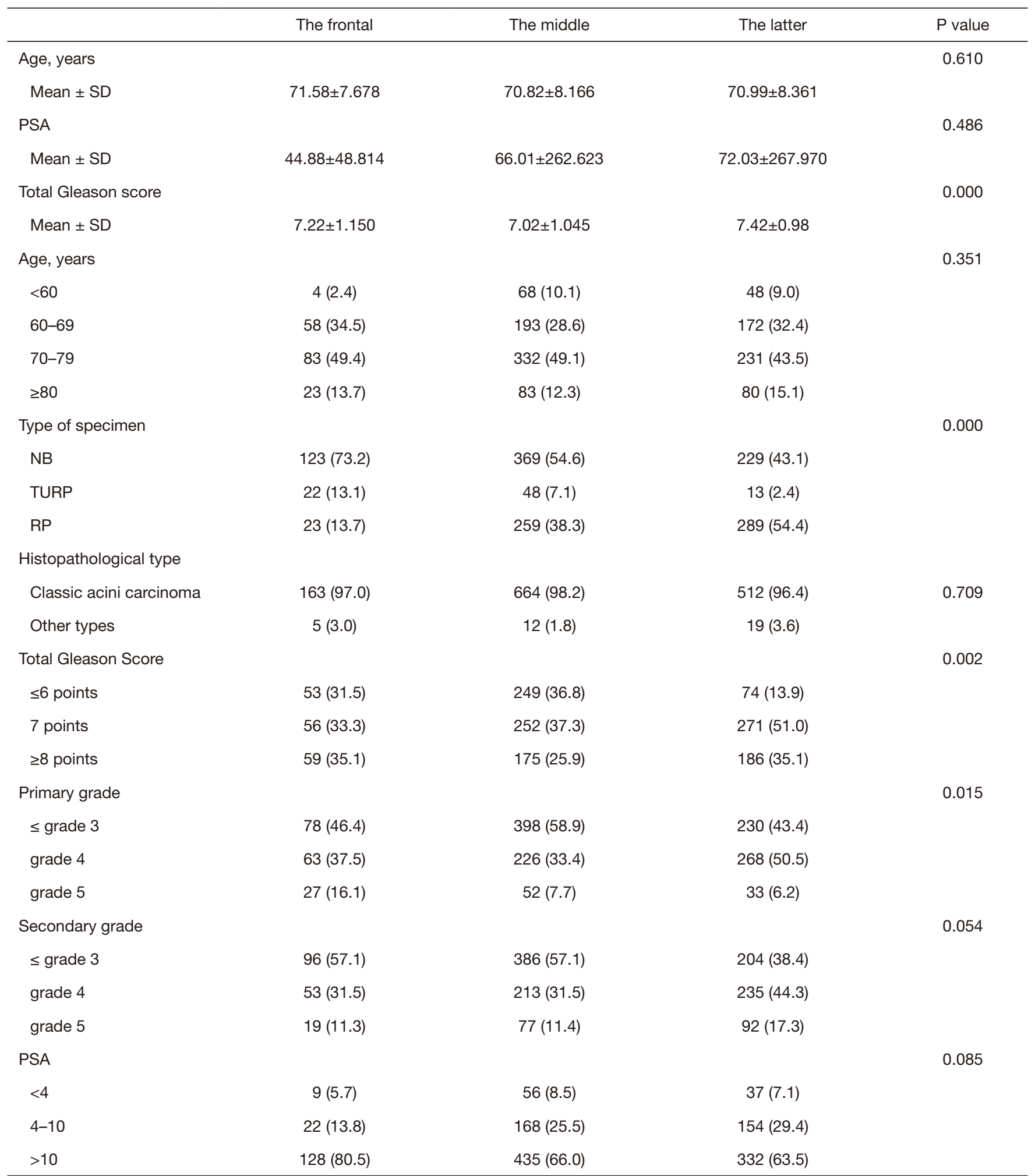

GS, Gleason score, NB, needle biopsy, TURP, transurethral resection of the prostate, RP, radical prostatectomy, prostate-specific antigen. 
Table 2 Distribution of GS in age, type of specimen and histopathological type in three cohorts

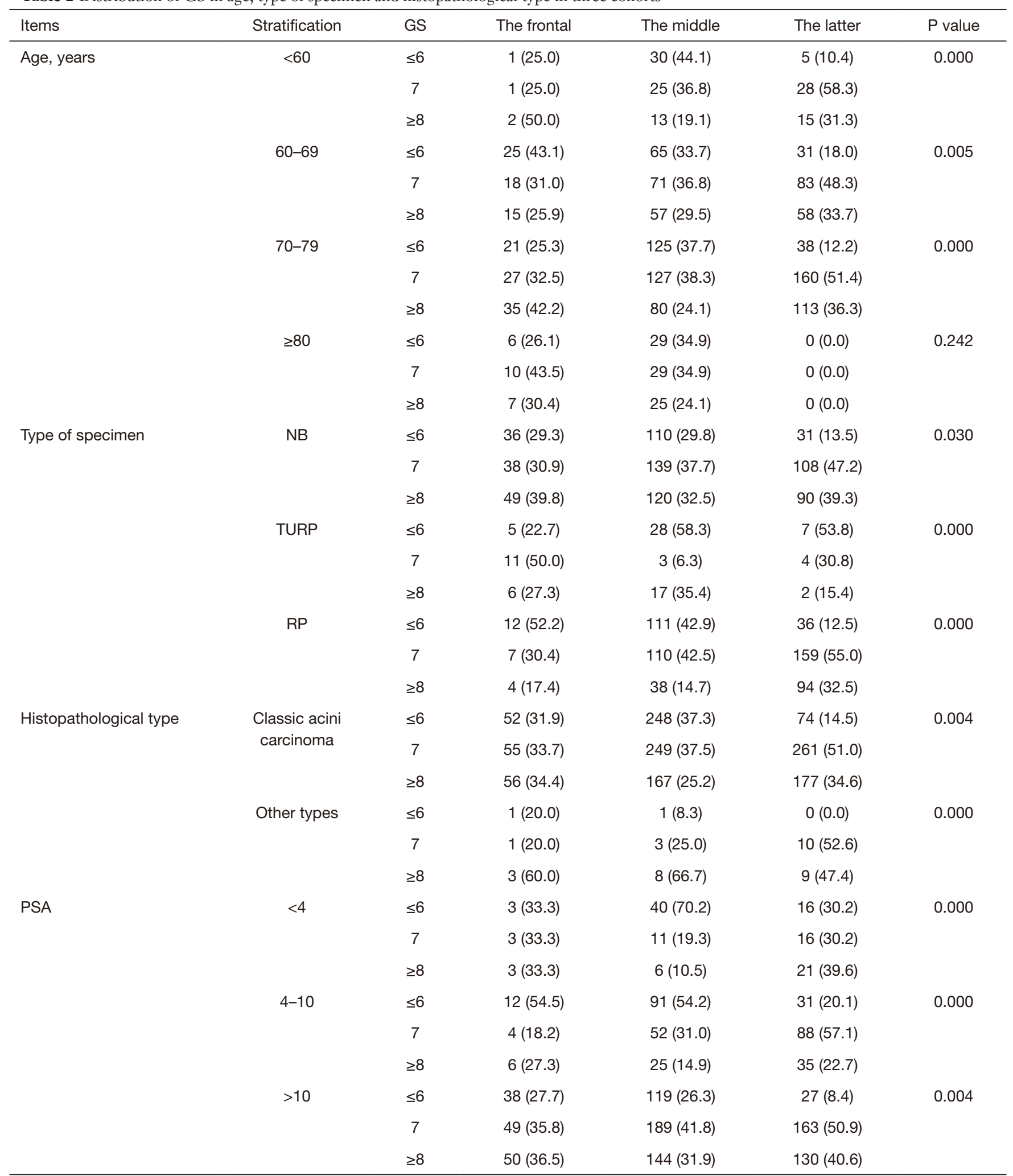

GS, Gleason score, NB, needle biopsy, TURP, transurethral resection of the prostate, RP, radical prostatectomy, prostate-specific antigen. 
Table 3 Trend of GS in age, type of specimen and histopathological type

\begin{tabular}{|c|c|c|c|c|c|}
\hline Items & Stratification & \multicolumn{4}{|c|}{ Total Gleason score } \\
\hline \multirow[t]{3}{*}{ Age, years } & $<60$ & $8.00 \pm 1.826$ & $6.93 \pm 1.097$ & $7.42 \pm 0.942$ & 0.016 \\
\hline & $60-69$ & $6.95 \pm 1.099$ & $7.09 \pm 1.055$ & $7.39 \pm 1.057$ & 0.005 \\
\hline & $70-79$ & $7.35 \pm 1.120$ & $6.96 \pm 0.992$ & $7.45 \pm 0.994$ & 0.000 \\
\hline \multirow[t]{3}{*}{ Type of specimen } & NB & $7.34 \pm 1.179$ & $7.21 \pm 1.080$ & $7.41 \pm 0.921$ & 0.058 \\
\hline & TURP & $7.14 \pm 0.889$ & $6.98 \pm 1.436$ & $6.85 \pm 1.281$ & 0.802 \\
\hline & $\mathrm{RP}$ & $6.65 \pm 1.071$ & $6.76 \pm 0.838$ & $7.45 \pm 1.006$ & 0.000 \\
\hline $\begin{array}{l}\text { Histopathological } \\
\text { type }\end{array}$ & Classic acini carcinoma & $7.21 \pm 1.159$ & $7.00 \pm 1.034$ & $7.40 \pm 0.978$ & 0.000 \\
\hline \multirow{2}{*}{ PSA } & $4-10$ & $6.91 \pm 1.231$ & $6.65 \pm 0.910$ & $7.18 \pm 0.937$ & 0.000 \\
\hline & $>10$ & $7.30 \pm 1.132$ & $7.23 \pm 1.056$ & $7.55 \pm 0.946$ & 0.000 \\
\hline
\end{tabular}

Table 4 Trend of primary grade in age, type of specimen and histopathological type

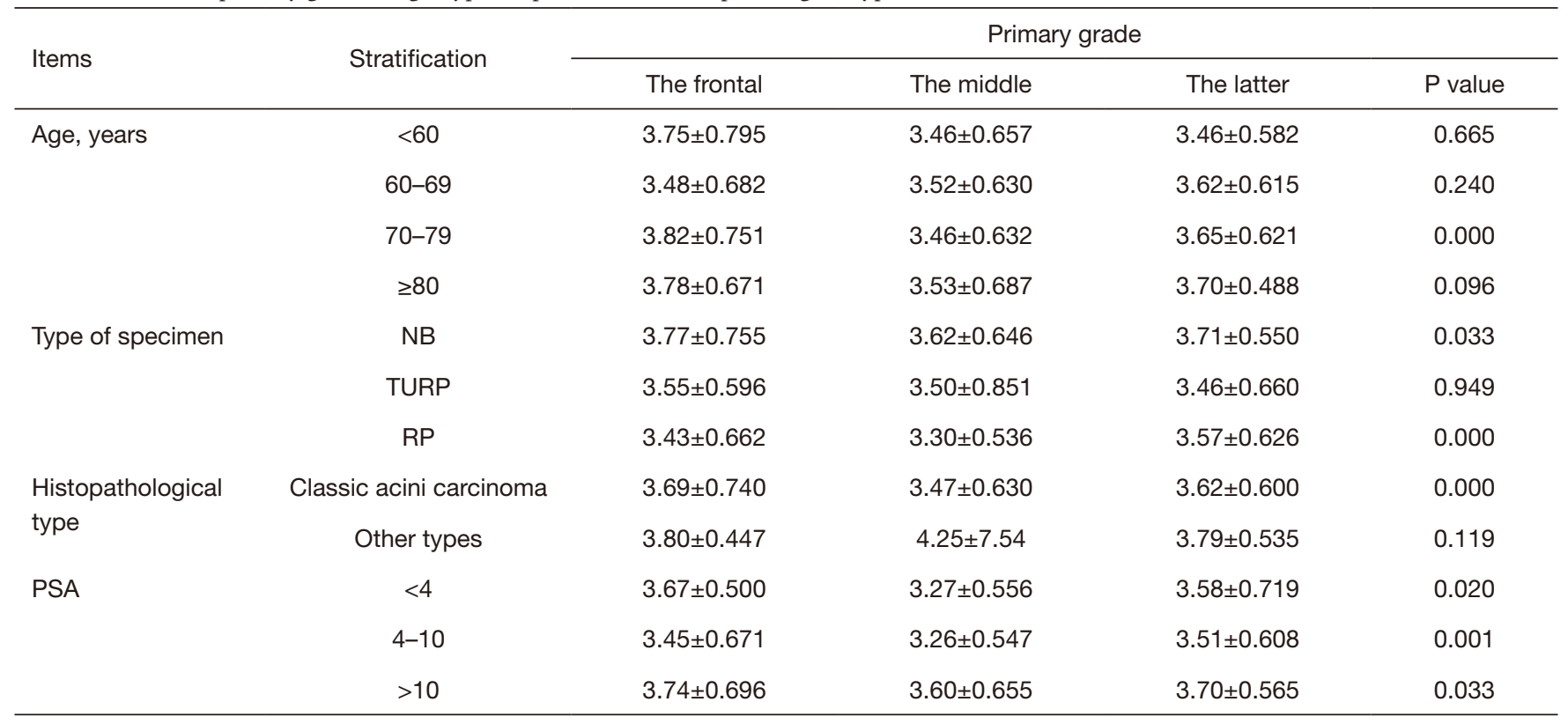

decreased from $31.5 \%$ in $1996-2006$ and $36.8 \%$ in 2007 2015 to $13.9 \%$ in 2016-2019, percentage of GS7 increased from $33.3 \%$ in $1996-2006$ and $37.3 \%$ in $2007-2015$ to $51.0 \%$ in 2016-2019, and percentage of GS $\geq 8$ remained constant. Average of GS increased from 7.22 in 1996-2006 and 7.02 in 2007-2015 to 7.42 in 2016-2019. In specimen from NB and RP, average of GS increased with significant statistical difference. Upward trend of GS in prostate cancer was observed in patients from our center in accord with previous studies.

Gleason score was defined 5 patterns according to the degree of differentiation. The largest area decided primary 
Table 5 Trend of secondary grade in age, type of specimen and histopathological type

\begin{tabular}{|c|c|c|c|c|c|}
\hline Items & Stratification & \multicolumn{4}{|c|}{ Second grade } \\
\hline \multirow[t]{3}{*}{ Age, years } & $<60$ & $3.504 .25 \pm 0.957$ & $3.47 \pm 0.680$ & $3.96 \pm 0.683$ & 0.000 \\
\hline & $60-69$ & $3.47 \pm 0.627$ & $3.56 \pm 0.720$ & $3.77 \pm 0.734$ & 0.003 \\
\hline & $70-79$ & $3.54 \pm 0.721$ & $3.51 \pm 0.671$ & $3.80 \pm 0.671$ & 0.000 \\
\hline \multirow[t]{3}{*}{ Type of specimen } & NB & $3.58 \pm 0.724$ & $3.59 \pm 0.713$ & $3.70 \pm 0.675$ & 0.127 \\
\hline & TURP & $3.59 \pm 0.590$ & $3.48 \pm 0.743$ & $3.38 \pm 0.768$ & 0.692 \\
\hline & $\mathrm{RP}$ & $3.22 \pm 0.671$ & $3.47 \pm 0.666$ & $3.88 \pm 0.735$ & 0.000 \\
\hline $\begin{array}{l}\text { Histopathological } \\
\text { type }\end{array}$ & Classic acini carcinoma & $3.53 \pm 0.714$ & $3.53 \pm 0.696$ & $3.78 \pm 0.713$ & 0.000 \\
\hline \multirow{2}{*}{ PSA } & $4-10$ & $3.45 \pm 0.739$ & $3.39 \pm 0.619$ & $3.67 \pm 0.657$ & 0.001 \\
\hline & $>10$ & $3.56 \pm 0.696$ & $3.63 \pm 0.723$ & $3.85 \pm 0.739$ & 0.000 \\
\hline
\end{tabular}

grade while the second decided secondary grade, and the sum was GS (18). Primary grade is the most important factor influencing GS and secondary grade less. The past literatures always focused on GS but lacked study on primary and secondary grade. Proportion of GS $\leq 3$ decreased to $43.4 \%$ in 2016-2019, and proportion of 4 increased to $50.5 \%$. That means PCa of primary grade $\leq 3$ was diagnosed less and less while GS 4 more and more. The change is most responsible to the change of GS mentioned above. The GS of secondary grade remained stable. Secondary grade affected GS less than primary grade. Primary grade is the main factor influencing GS change followed by secondary grade.

GS changed with type of specimen. Proportion of GS $\leq 6,7$ and $\geq 8$ in NB accounted for $41 \%, 48.9 \%$ and $10.1 \%$, respectively (10); $21.5 \%, 68.8 \%$ and $9.7 \%$ respectively in RP (19); $81.4 \%, 14.1 \%$ and $4.5 \%$ respectively in TURP (20). Mehta et al. (21) reported that $32.7 \%$ cases with GS 6 in NB turned out to 7 or more in RP. From that, GS in TURP is lowest and NB lower than RP. According to the distribution of specimen in our research, proportion of NB and TURP inclined while RP increased. It was speculated that GS in our research may go up. Average GS of RP increased from 6.65 in 1996-2006 and 6.76 in 20072015 to 7.45 in 2016-2019, which was in correspondence with our speculation. Results in RP specimen explained reasonable to this situation, showed that proportion of GS $\leq 6$ and 7 decreased and $\geq 8$ increased, in other words, GS had a upward trend. Percentage of GS $\leq 6$ in NB decreased from $29.3 \%$ in $1996-2006$ and 29.8 in $2007-2015$ to 13.5 in 2016-2019, GS 7 increased from $30.9 \%$ in 1996-2006 and $37.7 \%$ in $2007-2015$ to $47.2 \%$ in $2016-2019$, and GS $\geq 8$ almost remained stable, that meant a upward trend of GS. It was corresponded to GS changing trend after 2006 reported by literature. In Treurniet's opinion, GS in RP was lower than that in NB (22). Average of RP was higher than NB and TURP in 2016-2019, and the proportion of RP raised to $54.4 \%$, resulting in elevation of GS. GS of TURP was lower and reduction of sum contributed to GS upward trend to a certain extent. It was consistent with literatures (8).

There was no significantly difference of GS between age $\leq 55$ year old and $>56$ found by Billis (23). Richstone (24) reported that mean GS of age $\geq 70$ turned out higher than $<70$. Alibhai (15) held opinion that GS went up by age. With results from the present study, average of age and age distribution remained fairly static throughout three cohorts, but after segmented and stratified by age every ten years, difference emerged. Proportion of GS $\leq 6$ decreased in patients below 80 years in 2016-2019 compared with 1996-2006 and 2007-2015. In every age stage below 80, the average of GS increased significantly. The population ageing was not responsible to the upward shifting according to the present study.

Difference of histopathological type distribution 
in various times may lead to change of GS. Now that prostatic ductal carcinoma Gleason score was $4+4=8$ (10). Hanses (16) held opinion that sarcomatoid carcinoma was a high-level cancer with GS ranking 7 to 10 . Change of histopathological type may result in change of GS. PCa GS varied from histopathological type. Classic acinar carcinoma occupied a dominant position in our research, the other types less and affected less. Proportion of classic acinar carcinoma decreased, and the same change took place in mean of GS, primary and secondary grade. As for GS distribution, no significant diversification in GS $\leq 6,7$ and $\geq 8$. Different histopathological type influenced change of GS just a little.

Zivković et al. (25) believed PSA had a close relationship with GS. Gofrit et al. (17) reported that serum PSA could predict GS. Serum PSA more than $12 \mathrm{ng} / \mathrm{mL}$ in NB predicted $62 \% \mathrm{PCa}$, and less than $12 \mathrm{ng} / \mathrm{mL} 18 \% \mathrm{PCa}$. Elevated serum PSA was obeserved year by year. Patients with serum PSA in the gray zone and GS over 6 occupied $79.8 \%$ in 2016-2019 compared with 45.5\% in 1996-2006 and $45.9 \%$ in $2007-2015$. And patients with serum PSA over $10 \mathrm{ng} / \mathrm{mL}$ and GS over 6 occupied $91.5 \%$ in 2016 2019 compared with $72.3 \%$ in $1996-2006$ and $73.7 \%$ in 2007-2015. GS in this two groups increased significantly $(\mathrm{P}<0.05)$.

Change of diagnostic criteria may lead to change of GS (13). ISUP system was recommended for Gleason score after 2005 and modify system was used before 2005. Main difference between ISUP system and modify system: Branch shape or size changes remarkably complex glands, small lumens dysplastic glands to modify the system definition level 3 cancer, ISUP system level 4 carcinoma. These changes caused quantity of level 3 cancer got less while level 4 more $(10,26)$, and proportion of GS 6 went down while GS 7 went up. That's might be the main reason of GS increasing after 2005.

In summary, primary grade, age, serum PSA and specimen type were the main reasons for GS changing while secondary grade, tissue types and diagnostic criteria influenced less. PCa incidence was relatively low in China, and study on PCa started lately. After efforts of 20 years, great progress was made by application of serum PSA and prostate biopsy in PCa screening, and early diagnosis and treatment of prostate had gotten some progress in China. Change of GS would impact on treatment of PCa. Clinicians should square up the change combined with other clinical and radiological data, provide chance of operation to more patients and improve the prognosis of
PCa patients.

\section{Conclusions}

Our research found the upward changing trend of PCa GS. However, there were also shortage in our research, such as lack of complete follow-up data. We are going to collect follow-up data in the next step and observe the relationship of GS change and prognosis. Tumor heterogeneity should be discussed in next step.

\section{Acknowledgments}

Funding: This work was supported by the Prostate Cancer Foundation (13YOUN14) with Prof. Hu Hao as the recipient, and Peking University People's Hospital Research and Development Funds (RDY2019-19) with Zhang Weiyu as the recipient.

\section{Footnote}

Reporting Checklist: The authors have completed the STROBE reporting checklist. Available at http://dx.doi. org/10.21037/gs-20-659

Data Sharing Statement: Available at http://dx.doi. org/10.21037/gs-20-659

Conflicts of Interest: All authors have completed the ICMJE uniform disclosure form (available at http://dx.doi. org/10.21037/gs-20-659). The authors have no conflicts of interest to declare.

Ethical Statement: The authors are accountable for all aspects of the work in ensuring that questions related to the accuracy or integrity of any part of the work are appropriately investigated and resolved. The study was conducted in accordance with the Declaration of Helsinki (as revised in 2013). The study was approved by the local ethics committee of Peking University People's Hospital (PKUPH) (No. 2017PHB143) and individual consent for this retrospective analysis was waived.

Open Access Statement: This is an Open Access article distributed in accordance with the Creative Commons Attribution-NonCommercial-NoDerivs 4.0 International License (CC BY-NC-ND 4.0), which permits the noncommercial replication and distribution of the article with 
the strict proviso that no changes or edits are made and the original work is properly cited (including links to both the formal publication through the relevant DOI and the license). See: https://creativecommons.org/licenses/by-nc-nd/4.0/.

\section{References}

1. Chen W, Zheng R, Baade P, et al. Cancer Statistics in China, 2015. Ca Cancer J Clin 2016;66:115-32.

2. Epstein JI, Egevad L, Amin MB, et al. The 2014 International Society of Urological Pathology (ISUP) Consensus Conference on Gleason Grading of Prostatic Carcinoma: Definition of Grading Patterns and Proposal for a New Grading System. Am J Surg Pathol 2016;40:244-52.

3. Epstein JI, Allsbrook WC Jr, Amin MB, et al. The 2005 International Society of Urological Pathology (ISUP) Consensus Conference on Gleason Grading of Prostatic Carcinoma. Am J Surg Pathol 2005;29:1228-42.

4. Sathianathen NJ, Konety BR, Crook J, et al. Landmarks in prostate cancer. Nat Rev Urol 2018;15:627-42.

5. Delahunt B, Miller RJ, Srigley JR, et al. Gleason grading: past, present and future. Histopathology 2012;60:75-86.

6. Iczkowski KA, Lucia MS. Current perspectives on Gleason grading of prostate cancer. Curr Urol Rep 2011;12:216-22.

7. Pierorazio PM, Walsh PC, Partin AW, et al. Prognostic Gleason grade grouping: data based on the modified Gleason scoring system. BJU Int 2013;111:753-60.

8. Gilliland FD, Gleason DF, Hunt WC, et al. Trends in Gleason score for prostate cancer diagnosed between 1983 and 1993. J Urol 2001;165:846-50.

9. Chism DB, Hanlon AL, Troncoso P, et al. The Gleason score shift: score four and seven years ago. Int $\mathrm{J}$ Radiat Oncol Biol Phys 2003;56:1241-7.

10. Zareba P, Zhang J, Yilmaz A, et al. The impact of the 2005 International Society of Urological Pathology (ISUP) consensus on Gleason grading in contemporary practice. Histopathology 2009;55:384-91.

11. Danneman D, Drevin L, Robinson D, et al. Gleason Inflation 1998-2011: a registry study of 97168 men. BJU Int 2015;115:248-55.

12. Bell KJ, Del Mar C, Wright G, et al. Prevalence of incidental prostate cancer: A systematic review of autopsy studies. Int J Cancer 2015;137:1749-57.

13. Uemura H, Hoshino K, Sasaki T, et al. Usefulness of the 2005 International Society of Urologic Pathology
Gleason grading system in prostate biopsy and radical prostatectomy specimens. BJU Int 2009;103:1190-4.

14. Billis A, Guimaraes MS, Freitas LL, et al. The impact of the 2005 international society of urological pathology consensus conference on standard Gleason grading of prostatic carcinoma in needle biopsies. J Urol 2008; 180:548-52.

15. Alibhai SM, Krahn MD, Fleshner NE, et al. The association between patient age and prostate cancer stage and grade at diagnosis. BJU Int 2004;94:303-6.

16. Hansel DE, Epstein JI. Sarcomatoid carcinoma of the prostate: a study of 42 cases. Am J Surg Pathol 2006;30:1316-21.

17. Gofrit ON, Zorn KC, Taxy JB, et al. Predicting the risk of patients with biopsy Gleason score 6 to harbor a higher grade cancer. J Urol 2007;178:1925-8.

18. Humphrey PA, Moch H, Cubilla AL, et al. The 2016 WHO Classification of Tumours of the Urinary System and Male Genital Organs-Part B: Prostate and Bladder Tumours. Eur Urol 2016;70:106-19.

19. Corcoran NM, Hong MK, Casey RG, et al. Upgrade in Gleason score between prostate biopsies and pathology following radical prostatectomy significantly impacts upon the risk of biochemical recurrence. BJU Int 2011;108:E202-10.

20. Lee DH, Chung DY, Lee KS, et al. Clinical experiences of incidental prostate cancer after transurethral resection of prostate (TURP) according to initial treatment: a study of a Korean high volume center. Yonsei Med J 2014;55:78-83.

21. Mehta V, Rycyna K, Baesens BM, et al. Predictors of Gleason Score (GS) upgrading on subsequent prostatectomy a single Institution study in a cohort of patients with GS 6. Int J Clin Exp Pathol 2012;5:496-502.

22. Treurniet KM, Trudel D, Sykes J, et al. Downgrading of biopsy based Gleason score in prostatectomy specimens. J Clin Pathol 2014;67:313-8.

23. Billis A, Magna LA, Lira MM, et al. Relationship of age to outcome and clinicopathologic findings in men submitted to radical prostatectomy. Int Braz J Urol 2005;31:534-9.

24. Richstone L, Bianco FJ, Shah HH, et al. Radical prostatectomy in men aged >or $=70$ years: effect of age on upgrading, upstaging, and the accuracy of a preoperative nomogram. BJU Int 2008;101:541-6.

25. Zivković S, Velicković L, Kostov M, et al. Relation of serum prostate-specific antigen and the Gleason grade 
in patients with prostatic carcinoma. Vojnosanit Pregl 2003;60:449-54.

26. Dong F, Wang C, Farris AB, et al. Impact on the Clinical

Cite this article as: Zhang W, Wang G, Lan F, Wang H, Shen D, Xu K, Xu T, Hu H. Exploration on Gleason score variation trend of patients with prostate carcinoma from 1996 to 2019: a retrospective single center study. Gland Surg 2021;10(2):607-617. doi: 10.21037/gs-20-659
Outcome of Prostate Cancer by the 2005 International Society of Urological Pathology Modified Gleason Grading System. Am J Surg Pathol 2012;36:838-43. 
A

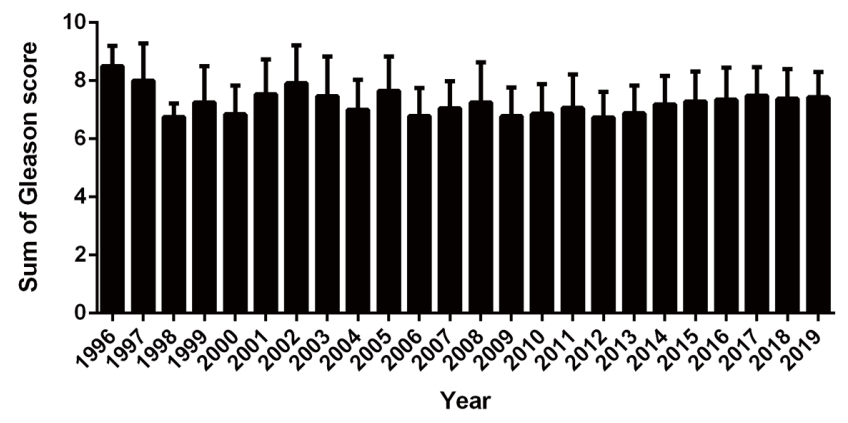

C

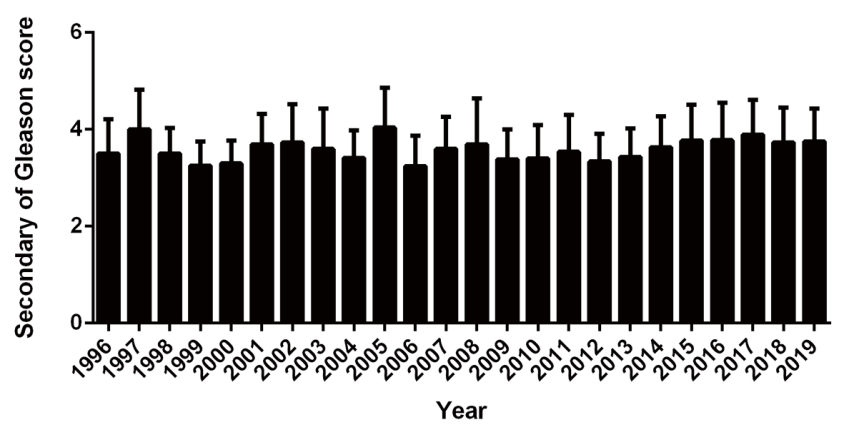

B

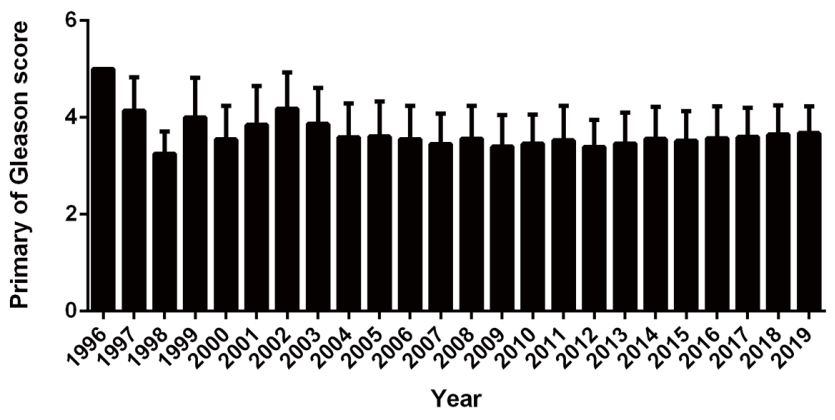

Figure S1 The total score, primary and secondary grade of Gleason floated by year. Several peaks and valleys shifted effected by factors, such as the application of PSA, the publication of China Urology Association guidelines, the increasing medical examination. 